\title{
SINGULARITIES IN MASS-LOADED MHD FLOW: THE COMETARY BOW SHOCK
}

\author{
I. Kh. Khabibrakhmanov, ${ }^{1}$ A. J. Coates, ${ }^{2}$ and V. L. Galinsky ${ }^{1}$
}

Abstract. We present a one-dimensional model of the mass-loading of the solar wind by cometary ions which predicts a singularity in the mass-loaded flow at $\mathbf{M}=2$. Further, a subshock occurs when the flow speed reaches $M \approx 1.15$. The shape of the cometary bow shock in two dimensions is predicted, by requiring that the flow Mach number of the shock is 2 taking the velocity component normal to the shock surface. The Mach number results compare favourably with observations at comet Halley.

\section{Introduction}

Many of the essential features of supersonic mass loaded plasma flow can be established using the simple onedimensional hydrodynamic model of Biermann et al. [1967] (see also Galeev at al. [1985]). This model predicts that continuous stationary flow of the solar wind loaded by newborn cometary ions is possible only up to a "selfreversal" point where the local Mach number $M=1$, and where the mass flux of the loaded flow normalised to the mass flux of the solar wind at infinity reaches $4 / 3$. However, this result does not predict the actual position of the cometary bow shock. It shows that as the solar wind flow slows due to mass loading, a shock transition must occur before this critical point in the flow. Strictly, the possibility of a continuous transition across the sonic point of the mass loaded plasma flow is excluded. Numerical simulations [e.g., Schmidt and Wegmann, 1982, Baranov et al., 1986] of the solar wind interaction with comets have shown that the Mach number of the cometary bow shock is close to 2 in the subsolar region.

Hybrid simulations have been used to examine the structure of the cometary bow shock [e.g., Galeev and Lipatov, 1984, Omidi and Winske, 1987]. The results showed systems of steepening waves in the mass loaded flow rather than a sharp shock. The MHD approach used here gives the position of the shock within the cometary coma rather than the structure of the shock transition.

Huddleston et al [1990] have recently calculated the inner limit of the shock location as the locus of the selfreversal point in the flow, using a one-dimensional model extended to give a two-dimensional shape in the plane of the Giotto spacecraft trajectory and the Sun. They did not calculate the position of the shock itself.

The connection between the Mach number of the cometary bow shock and its spatial position relative to the cometary nucleus has not yet been established analytically. The cometary bow shock is characterized by the absence of a "piston" or "rigid obstacle" which is present

\footnotetext{
${ }^{1}$ Space Research Institute, USSR Academy of Sciences, Moscow, USSR

${ }^{2}$ Mullard Space Science Laboratory, University College London, UK

Copyright 1991 by the American Geophysical Union.

Paper number 91GL01560

0094-8534/91/91GL-01560\$3.00
}

in the case of the solar wind interaction with planets having a strong intrinsic magnetic field. At comets, information concerning the shock transition is created in the solar wind plasma flow itself during the mass loading process rather than by the boundary conditions. This statement will be our working hypothesis here.

The stationary solution was discussed by Galeev and Khabibrakhmanov [1990a] and Khabibrakhmanov and Verheest [1990], who showed from linear analysis of the local dispersion equation that the local increment (i.e., the growth rate of small disturbances at a given point in the flow) of magnetosonic waves changes its sign at the point in the flow where the Mach number equals 2. More recently it was shown [Galeev and Khabibrakhmanov, $1990 \mathrm{~b}, \mathrm{c}, \mathrm{d}]$ that this local positive increment is the result of wave steepening or a "gradient catastrophe" of the stationary solution.

\section{Overview of one-dimensional mass loading theory}

Mass loading of the solar wind by cometary ions can be described by a one-dimensional MHD system [Biermann et al., 1967, Galeev et al., 1985] which may be rewritten in characteristic form as follows [Galeev and Khabibrakhmanov, 1990b]:

$$
\begin{aligned}
\hat{D}_{ \pm} P \pm \rho c \hat{D}_{ \pm} u & =\frac{\nu m u}{2}((\gamma-1) u \mp 2 c) \\
\hat{D}_{0} P-c^{2} \hat{D}_{0} \rho & =\frac{\nu m}{2}\left((\gamma-1) u^{2}-2 c^{2}\right)
\end{aligned}
$$

where $\rho$ is the mass density, $u$ is the bulk velocity, $P=$ $\left(P_{t h}+B^{2} / 2 \mu_{o}\right)$ is the sum of the thermal pressure of the ions and the magnetic pressure, $B$ is the magnetic field, $m$ is the mass of the newborn cometary ions and $\nu$ is their production rate (ions $\mathrm{m}^{-3} \mathrm{~s}^{-1}$ ), $\gamma$ is the specific heat ratio of the plasma and the magnetosonic speed $c$ is defined by $c^{2}=\left(\gamma P_{t h}+B^{2} / \mu_{o}\right) / \rho$. Equation (1) describes the evolution of acoustic disturbances propagating along the acoustic characteristics $C_{ \pm}$with the corresponding differential operator $\hat{D}_{ \pm}=\partial / \partial t+(u \pm c) \partial / \partial x$. Equation (2) describes the propagation of entropy disturbances moving with the flow along the $C_{0}$ characteristic defined by the operator $\hat{D}_{0}=\partial / \partial t+u \partial / \partial x$. This system coincides with that of Galeev et al. [1985] except that time dependence is included here.

To analyse Equations (1) and (2) for discontinuities it is convenient to recast them in terms of the Riemann invariants of the flow:

$$
R_{ \pm}=u_{x} \pm c_{x} \pm \frac{1}{2 \rho} c \rho_{x}, \quad L=c_{x}-\frac{1}{2 \rho} c \rho_{x}
$$

where the subscript $x$ denotes the partial derivative with respect to $x$. We assume here that the plasma flow is perpendicular to the magnetic field, so that isotropization effects due to Alfvènic turbulence are excluded and $\gamma=2$. Applying the differential operator $\hat{D}_{x}=\partial / \partial x$ to each of the equations (1) and (2), one finds the following transport equations for the quantities in (3): 


$$
\begin{aligned}
\hat{D}_{ \pm} R_{ \pm}+\frac{3}{4} R_{ \pm}^{2}+\frac{1}{4} R_{ \pm} R_{\mp}+\frac{L}{4}( & \left.R_{ \pm}-R_{\mp}\right) \pm \Delta= \\
& \pm \hat{D}_{x}\left(\frac{\nu m u}{2 \rho c}(u \mp 2 c)\right), \\
\hat{D}_{0} L+\frac{3}{4} L\left(R_{+}+R_{-}\right)-\Delta= & \hat{D}_{x}\left(\frac{\nu m}{2 \rho c}\left(u^{2}-2 c^{2}\right)\right),
\end{aligned}
$$

where

$$
\Delta=\frac{\nu m}{8 \rho}\left[\left(2 L+R_{+}-R_{-}\right)+\frac{u^{2}-c^{2}}{c^{2}}\left(2 L-R_{+}+R_{-}\right)\right]
$$

To illustrate the existence of the gradient catastrophe in these equations, we consider first the case of no mass loading $(\nu=0)$ and isentropic flow $\left(c / \rho^{1 / 2}=\right.$ const and $L=0$ ). The first equation of system (4) is a Ricatti nonlinear equation having solutions which tend to infinity in a finite time. It can be transformed to a linear differential equation by the substitution $R_{ \pm}=1 / z$, giving:

$$
R_{+}=R_{+, 0}\left(\frac{c^{2} \rho}{c_{0}^{2} \rho_{0}}\right)^{\frac{1}{8}}\left(1+R_{+, 0} \frac{3}{4} \int_{0}^{t}\left(\frac{c^{2} \rho}{c_{0}^{2} \rho_{0}}\right)^{\frac{1}{8}} d t\right)^{-1}
$$

Here the integration is along the characteristic $C_{+}$and all initial values at the time $t=0$ are denoted by the subscript "0". One can see from Equation (6) that if the initial value $R_{+, 0}\left(R_{+, 0}=R_{+}(x, t=0)\right)$ is positive then $R_{+} \geq 0$ for all $t \geq 0$ and the plasma flow will remain continuous. However, if $R_{+, 0} \leq 0$ at some point $x_{0}$ along the characteristic $C_{+}$, then $R_{+} \leq 0$ everywhere except when the denominator in (5) is zero. At this point the gradient catastrophe occurs. The position of the gradient catastrophe is determined by the equation:

$$
\frac{3}{4} \int_{0\left(C_{+}\right)}^{t_{k}}\left(\frac{c^{2} \rho}{c_{0}^{2} \rho_{0}}\right)^{\frac{1}{8}} d t=-\frac{1}{R_{+, 0}} \quad\left(R_{+, 0}<0\right)
$$

For the time interval $t \geq t_{k}$ continuous plasma flow is not possible and dispersive effects can stop the gradient catastrophe in the flow. Similar calculations were used for the piston problem in a perfect gas (see for example the textbook by Landau and Lifshitz [1987]).

Returning to the case of mass loaded supersonic plasma flow $(\nu \neq 0)$, using the upper sign in Equation (1), for stationary flow the value of $R_{+}$may be written:

$$
(u+c) R_{+}=\frac{\nu m u}{2 \rho c}(u-2 c) .
$$

The value of $R_{+, 0}$ becomes zero $\left(R_{+, 0}\right.$ changes sign) at the point in the flow with Mach number $M=2$. According to the solution (5), any infinitesimal disturbance of the stationary flow will lead to the gradient catastrophe singularity here and the stationary solution of Galeev et al. [1985] is not possible for $M \leq 2$.

This conclusion does not depend on the mass loading profile. The source term $\nu$ may depend on the spatial position in the expanding neutral gas and on the flow parameters. Even if a stationary solution does not exist, a singularity of the plasma flow at $M=2$ is still present until the breakdown of the MHD description.

The characteristic spatial dimension of the collisionless shock is determined by the dispersion of the plasma. For slow motion of magnetized plasma exactly perpendicular to the magnetic field the dispersion of the magnetosonic waves was calculated in Khabibrakhmanov and Verheest [1990] by expansion of the solutions of the Vlasov equation using small parameters (ratios of characteristic spatial and time variations to the gyroradius and gyroperiod of the plasma particles). This method of expansion ignores the products of derivatives of the plasma flow parameters but takes into account the nonlinear variation of the flow parameters on scales larger than the gyroradius and gyroperiod. In the case of ions dominating the plasma thermal pressure, the dispersion parameter is:

$$
a_{D}^{2}=\frac{P}{4 \rho \Omega^{2}}\left\{1+\frac{3 P}{\rho u^{2}}\left[\frac{2 \Pi \rho}{m P^{2}}-3\right]\right\},
$$

here $\Omega$ is the gyrofrequency of the ions, dominated by the pickup ions, and $I$ is the second moment of the ion distribution function over the magnetic moment $\mu=v_{\perp}^{2} / 2 B$ of the particles (i.e., $\Pi=\int f \mu^{2} d^{3} \mathbf{v}$ ). For a Maxwellian distribution, the parameter $\lambda\left(=2 \Pi \rho / m P^{2}\right)$ is 4 , while for a ring distribution $\lambda=2$. For the mass loaded solar wind near the $M=2$ point one can find using an explicit expression for the distribution function of the ions [Galeev et al., 1985] that $\lambda=2.12$. This means that the dispersion length $a_{D}^{2}$ which is positive upstream of the cometary bow shock with Mach number $M=2$ can diminish to zero inside the shock. This happens at the point where the local Mach number decreases to $M_{c r}=\sqrt{1.32}=1.15$ (from equation (8)). At this point ion dispersion can no longer prevent the overturning of the plasma flow and an electron-proton subshock appears with dimensions much less than the ion dispersion length.

\section{Observations}

The Giotto plasma data set is, at least on the inbound pass, the most complete of any of the 1985-86 cometary probes, in that all the appropriate parameters (electrons, solar wind protons, magnetic field and cometary water group ions) were measured directly. Outbound only the electron parameters require assumptions. Recently Coates et al. [1990a] presented a refined set of these parameters and used them to perform a Rankine-Hugoniot jump analysis to determine the shock normals. This analysis was performed for two intervals, firstly well-upstream and downstream from the shock (between the points U2D2 in Figure 1 of Coates et al., [1990a]) and secondly immediately upstream and downstream (U1-D1) of the S1-S2 structure, which was interpreted as representing the shock itself. The different normals found using these intervals led to different shock Mach numbers as calculated by propagation into the upstream flow. These numbers were 1.03-1.14 for U1-D1 and 1.7-1.8 for U2-D2. For comparison to the theory in this paper, we interpret the U2-D2 transition as giving the Mach number of the entire shock structure and U1-D1 as giving the Mach number of the "subshock". Note that S2 was previously interpreted as a possible subshock [Coates et al., 1990a].

Using the measured parameters, Coates et al. [1990b] have calculated the magnetosonic Mach number of the flow in the shock region. Figure 1 shows the Mach number as a function of time and distance along the spacecraft track (this track was at $107^{\circ}$ to the comet-Sun line). The relevant features of this plot are that the U2 interval occurs at a flow Mach number $M \sim 2.5$ and the "subshock" (S2) appears at $M \sim 1.5$. The Mach number of the normal component (not shown here) is lower than that shown 


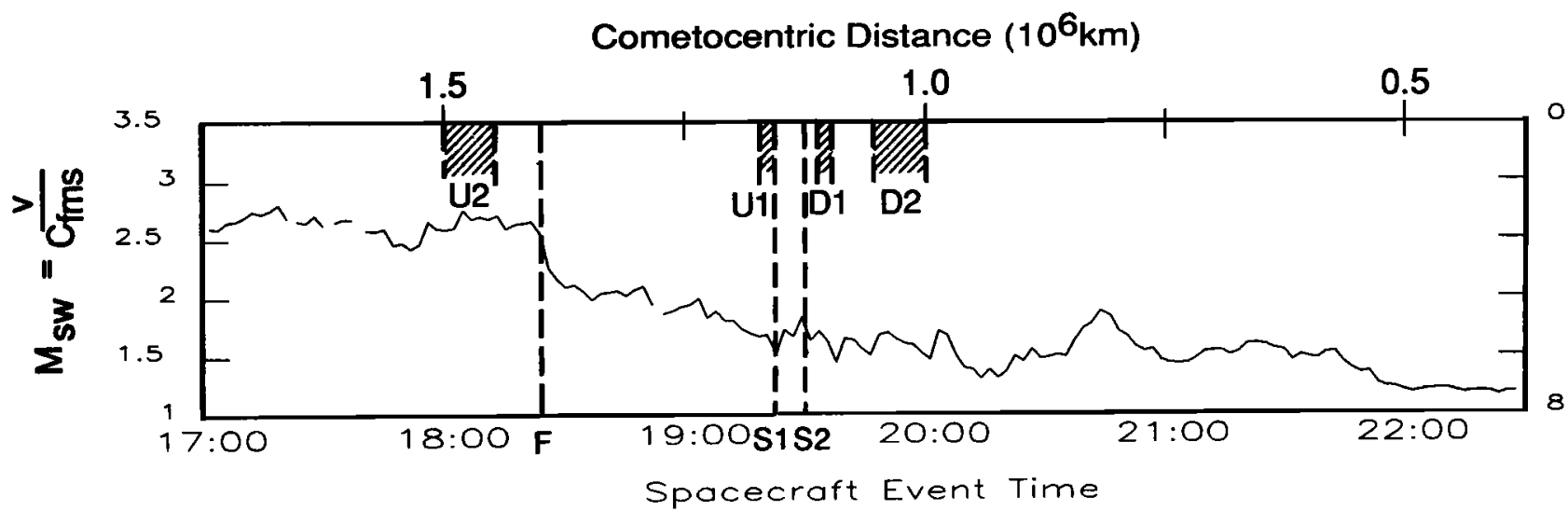

Fig. 1. Giotto measurements of the magnetosonic Mach number of the solar wind near the bow shock structure S1-S2. The upstream-downstream (U-D) averaging intervals used for the Rankine-Hugoniot analysis in Coates et al [1990a] immediately near (1) and well away from (2) the S1-S2 structure are shown (U1-D1 and U2-D2).

in Figure 1, and therefore closer to the predicted values, throughout this period. The precise value depends on which normal is taken [see Coates et al., 1990b], and consequently we show the magnitude here rather than any particular normal component. The Mach numbers for the ICE encounter with comet Giacobini-Zinner have also been published recently [Staines et al., 1991] and inspection of their results also shows that the Mach number at the shock is close to 2 .

\section{Discussion}

The observed Mach number of the quasiperpendicular bow shock was close to the predicted value of 2 and the subshock Mach number was close to the predicted 1.15. Therefore we may assume that the Mach number of the cometary bow shock calculated for the velocity component normal to the bow shock surface is equal to 2 around the shock surface: $M=2 / \sin \alpha$, where $\alpha$ is the angle between the solar wind velocity and the shock surface.

As can be seen from three dimensional simulations [Ogino et al., 1988] in the supersonic region the flow pattern is almost unidirectional. So one can calculate the local value of the mass flux from the continuity equation:

$$
\frac{d \rho u}{d x}=\frac{Q m}{4 \pi V_{g} \tau\left(x^{2}+y^{2}\right)} \exp \left(-\frac{\sqrt{x^{2}+y^{2}}}{V_{g} \tau}\right),
$$

where the source term is defined by $N\left(=Q / 4 \pi V_{g} \tau\right)$ and $V_{g}$, the density and velocity of the neutral gas, and by the photoionization time $\tau$.

Finally we obtain the equation defining the bow shock shape in cylindrical coordinates $(r, \theta)$ :

$$
\begin{gathered}
\frac{\rho u}{\rho_{\infty} u_{\infty}}=\frac{8 \gamma^{2}\left[\sin ^{2} \alpha+2(\gamma-1)\right]}{(\gamma-1)\left(\sin ^{2} \alpha+4 \gamma\right)^{2}}= \\
1+\frac{R_{L}}{r \sin \theta} \int_{0}^{\theta_{s h}} d \theta \exp \left(-\frac{y}{V_{g} \tau \sin \theta}\right),
\end{gathered}
$$

here $y=r \sin \theta=$ const along the flow line.

The solutions of this equation are shown in Figure 2 for two limiting cases: the adiabatic approximation $(\gamma=2)$ in the upper half and strong isotropization of new cometary ions $(\gamma=5 / 3)$ in the lower half. The outermost shapes are the limiting solutions for small values of the parameter $\beta=R_{L} / V_{g} \tau$, which determines the strength of the exponential decrease of the neutral gas density with distance $R_{L}=\left(Q m / 4 \pi V_{g} \tau \rho_{\infty} u_{\infty}\right)$. Other curves correspond to larger values of $\beta$ as indicated. In Table 1 we give the calculated distances $R_{s}$, where the shock appears along the stagnation line, for given values of $\beta$ using the parameters relevant to the Giotto encounter: $Q=10^{30}$ $\mathrm{s}^{-1}, V_{g} \tau=1.116 \cdot 10^{6} \mathrm{~km}, n_{\infty} u_{\infty}=2.266 \cdot 10^{18} \mathrm{~km}^{-2} \mathrm{~s}^{-1}$, $u_{\infty}=366 \mathrm{kms}^{-1}$ [Huddleston et al., 1990]. Note that

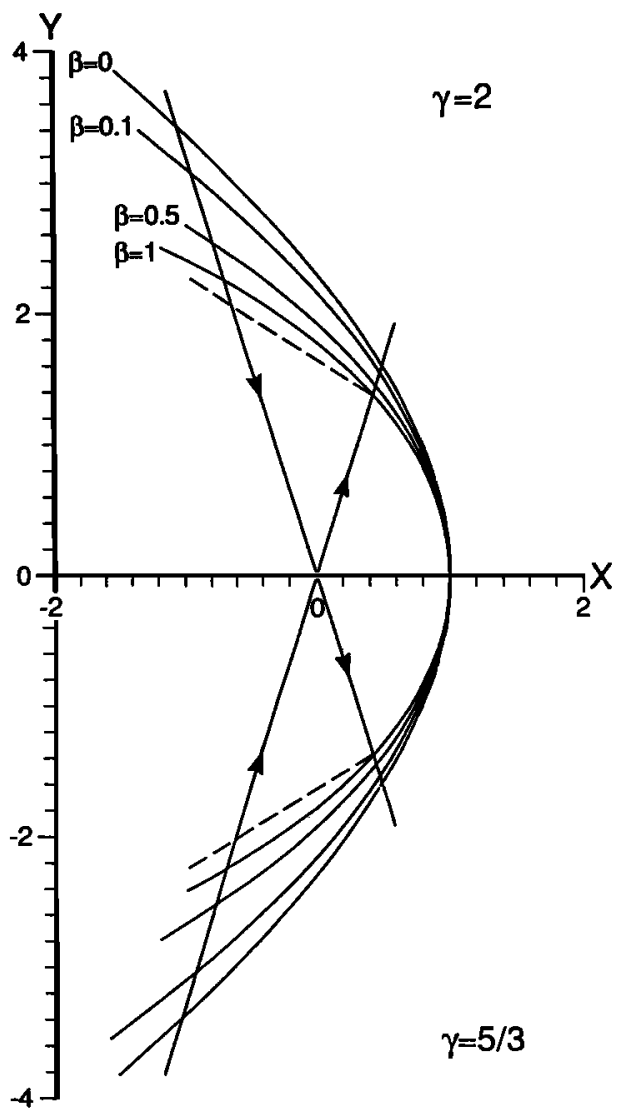

Fig. 2. Solutions of equation (12) for $\gamma=2$ (top panel) and $\gamma=5 / 3$ (bottom panel), for different values of the parameter $\beta$, and normalised to $R_{s}=1$ (see text). The lines with arrows show the angles of the Giotto trajectory and the dotted line shows the distance ratio of the observed bow shock crossings. 
TABLE 1. Dependence of the subsolar standoff distance $R_{s}$ on the parameter $\beta$ (see text)

\begin{tabular}{ccc}
\hline$\beta$ & $R_{s}(\mathrm{~km})[\gamma=2]$ & $R_{s}(\mathrm{~km})[\gamma=5 / 3]$ \\
\hline 0.1 & $2.21 \times 10^{6}$ & $1.27 \times 10^{6}$ \\
0.5 & $9.99 \times 10^{5}$ & $5.72 \times 10^{5}$ \\
1.0 & $4.54 \times 10^{5}$ & $2.60 \times 10^{5}$ \\
\hline
\end{tabular}

these values of the parameters would give $\beta \sim 0.5$, which with $\gamma=2$ gives a subsolar standoff distance of almost $10^{6} \mathrm{~km}$ and a flaring factor of approximately 2 , both of which are larger than those inferred from observations of the shock crossings (see below).

Another way of comparing with observations is to use a feature of the shape which is independent of the spatial scaling factor, assuming that the solar wind conditions are constant during the flyby. We have tried the ratio of the cometocentric distances of the observed shock crossings on the inbound and outbound legs of the spacecraft trajectory. For Giotto this ratio was close to 1.5. The trajectory is overlaid on the dimensionless coordinates in the two panels of Figure 2: the ratio of 1.5 corresponds to a straight line of gradient $-1 /\left(5 \tan 17^{\circ}\right)=-0.65$ on this plot. Lines with this gradient are shown dotted from the intersection of the $\beta=1$ solution with the outbound trajectory on each panel. This shows that a value of $\beta$ just greater than 1 would give a flaring factor which would fit theory and observations. $\beta \leq 1$ clearly gives too large a flaring factor. From Table 1 we note that the $\gamma=2$ case gives $R_{s}=4.54 \times 10^{5} \mathrm{~km}$ for $\beta=1$. This value is agreeably close to the values of $R$, found in two other papers: (1) the self-reversal point in the mass-loading model of $\mathrm{Hud}$ dleston et al. [1990] $\left(5.5 \times 10^{5} \mathrm{~km}\right)$ and (2) a paraboloid fit to the observations ([Coates et al., 1990a] $-5.98 \times 10^{5} \mathrm{~km}$ ).

However, the $\beta$ value determined using the parameters inferred by Huddleston et al [1990], $\beta \sim 0.5$, is inconsistent with the value $(\beta>1)$ that we infer here from comparing the $M=2$ curve with observations. A reason could be that different values of $\gamma$ are appropriate to the inbound (quasiperpendicular, $\gamma=2$ ) and outbound (quasiparallel, $\gamma=5 / 3$ ) cases, which have different scalings for a particular $\beta$. Another reason may be our assumption of exactly perpendicular flow and magnetic field.

Acknowledgments. AJC is grateful to the Royal Society, UK for support, and to IKI, USSR, for kind hospitality during the visit which made this collaboration possible.

\section{References}

Baranov, V. B., N. A. Zaitzev and M. G. Lebedev, Model of the interaction of solar wind with the atmosphere of comets, Sov. Astron., 63, 170-180, 1986. (In Russian).

Biermann, L., B. Brosowski and H. U. Schmidt, The interaction of the solar wind with a comet, Solar Phys., $1,254-283,1967$.

Coates, A. J. et al., Plasma parameters near the comet Halley bow shock, J. Geophys. Res., 95, 20,701-20,716, 1990 a.

Coates, A. J., et al., Plasma parameters near the comet Halley bow shock, Adv. Space Res., in press, $1990 \mathrm{~b}$.
Galeev, A. A., T. E. Cravens and T. I. Gombosi, Solar wind stagnation near comets, Astrophys. J., 289, 807819, 1985.

Galeev, A. A. and I. Kh. Khabibrakhmanov, Mach number of a cometary bow shock, Sov. Astron. Lett., 16, 200-202, 1990a.

Galeev, A. A. and I. Kh. Khabibrakhmanov, Dynamics of supersonic mignetized plasma flow with mass loading, Adv. Space Res., in press, $1990 \mathrm{~b}$.

Galeev, A. A. and I. Kh. Khabibrakhmanov, Gradient catastrophe of the supersonic mass-loaded magnetized plasma flow and the formation of bow shock, Proc. International Workshop on "Plasma Astrophysics", Eur. Space Agency Spec. Publ., SP-311, 95-98, 1990c.

Galeev, A. A. and I. Kh. Khabibrakhmanov, Gradient catastrophe of a loaded supersonic magnetized plasma flow and shock wave formation, Sov. Phys. JETP, 11, 916-920, 1990d.

Galeev, A. A. and A. S. Lipatov, Plasma processes in cometary atmospheres, Adv. Space Res., 4, no. 9, 229237, 1984.

Galeev, A. A., A. S. Lipatov and R. Z. Sagdeev, Numerical simulations of shock waves near comets: Structural features and energy dissipation mechanisms, Sov. Phys. JETP, 62, 866-871, 1985.

Huddleston, D. E., A. D. Johnstone and A. J. Coates, Determination of comet Halley gas emission characteristics from mass loading of the solar wind, $J$. Geophys. Res., 95, 21-30, 1990.

Khabibrakhmanov, I. Kh. and F. Verheest, Nonlinear supersonic motions of magnetized plasma flow with mass loading, J. Geophys. Res., 95, 10449-10457, 1990.

Landau, L. D. and E. M. Lifshitz, Fluid mechanics, second English edition, Pergamon, Oxford, 1987.

Ogino, T., R. J. Walker and M. Ashour-Abdalla, A three dimensional MHD model simulation of the solar wind with comet Halley, J. Geophys. Res., 99, 9568-9576, 1988.

Omidi, N., and D. Winske, A kinetic study of solar wind mass loading and cometary bow shocks, J. Geophys. Res., 92, 13,409-13,426, 1987.

Schmidt, H. U. and R. Wegmann, Plasma flow and magnetic fields in comets, in Comets, ed. L. L. Wilkening, Univ. of Arizona Press, Tucson, 538-560, 1981.

Staines, K. et al., Cometary water group ions in the region surrounding comet Giacobini-Zinner: distribution functions and bulk parameter estimates, Planet. Space Sci., 99, 479-506, 1991.

V.L. Galinsky and I.Kh. Khabibrakhmanov, Space Research Institute, USSR Academy of Sciences, Profsoyusnaya $84 / 32,117810$, Moscow, USSR

A.J. Coates, Mullard Space Science Laboratory, University College London, Holmbury St Mary, Dorking, Surrey RH5 6NT, UK

(Received 26 March, 1991

revised 10 May, 1991 accepted 28 May, 1991) 